\title{
Interactive Fuzzy Programming for Random Fuzzy Two-Level Integer Programming Problems through Fractile Criteria with Possibility
}

\author{
Masatoshi Sakawa, Takeshi Matsui \\ Department of System Cybernetics, Graduate School of Engineering, Hiroshima University, Higashi-Hiroshima, Japan \\ Email: sakawa@hiroshima-u.ac.jp,tak-matsui@hiroshima-u.ac.jp
}

Received April 17, 2013; revised May 17, 2013; accepted May 24, 2013

Copyright (C) 2013 Masatoshi Sakawa, Takeshi Matsui. This is an open access article distributed under the Creative Commons Attribution License, which permits unrestricted use, distribution, and reproduction in any medium, provided the original work is properly cited.

\begin{abstract}
This paper considers two-level integer programming problems involving random fuzzy variables with cooperative behavior of the decision makers. Considering the probabilities that the decision makers' objective function values are smaller than or equal to target variables, fuzzy goals of the decision makers are introduced. Using the fractile criteria to optimize the target variables under the condition that the degrees of possibility with respect to the attained probabilities are greater than or equal to certain permissible levels, the original random fuzzy two-level integer programming problems are reduced to deterministic ones. Through the introduction of genetic algorithms with double strings for nonlinear integer programming problems, interactive fuzzy programming to derive a satisfactory solution for the decision maker at the upper level in consideration of the cooperative relation between decision makers is presented. An illustrative numerical example demonstrates the feasibility and efficiency of the proposed method.
\end{abstract}

Keywords: Two-Level Integer Programming; Random Fuzzy Programming; Possibility; Fractile Criteria; Interactive Fuzzy Programming

\section{Introduction}

Decision making problems in hierarchical managerial or public organizations are often formulated as two-level mathematical programming problems $[1,2]$. In the context of two-level programming, the decision maker at the upper level first specifies a strategy, and then the decision maker at the lower level specifies a strategy so as to optimize the objective with full knowledge of the action of the decision maker at the upper level. In conventional multi-level mathematical programming models employing the solution concept of Stackelberg equilibrium, it is assumed that there is no communication among decision makers, or they do not make any binding agreement even if there exists such communication [1,3-5]. Compared with this, for decision making problems in such as decentralized large firms with divisional independence, it is quite natural to suppose that there exists communication and some cooperative relationship among the decision makers [2].

For two-level linear programming problems or multilevel ones such that decisions of decision makers in all levels are sequential and all of the decision makers essentially cooperate with each other, Lai [6] and Shih et al. [7] proposed fuzzy interactive approaches. In their methods, the decision makers identify membership functions of the fuzzy goals for their objective functions, and in particular, the decision maker at the upper level also specifies those of the fuzzy goals for the decision variables. The decision maker at the lower level solves a fuzzy programming problem with a constraint with respect to a satisfactory degree of the decision maker at the upper level. Unfortunately, there is a possibility that their method leads a final solution to an undesirable one because of inconsistency between the fuzzy goals of the objective function and those of the decision variables. In order to overcome the problem in their methods, by eliminating the fuzzy goals for the decision variables, Sakawa et al. have proposed interactive fuzzy programming for two-level or multi-level linear programming problems to obtain a satisfactory solution for decision makers [8,9]. Extensions to two-level linear fractional programming problems [10], decentralized two-level linear programming problems $[11,12]$, two-level linear fractional programming prob- 
lems with fuzzy parameters [13], and two-level noncomvex programming problems with fuzzy parameters [14] were provided. Further extensions to two-level linear programming problems with random variables, called stochastic two-level linear programming problems $[15,16]$, two-level integer programming problems [17], and twolevel linear programming problems involving fuzzy random variables, called fuzzy random two-level programming problems $[18,19]$, have also been considered. It should be noted here that fuzzy random variables [20-22] are considered to be random variables whose realized values are not real values but fuzzy numbers or fuzzy sets. Arecent survey paper of Sakawa and Nishizaki [23] is devoted to reviewing and classifying the numerous major papers in the area of so-called cooperative multilevel programming.

On the other hand, from a viewpoint of ambiguity and randomness different from fuzzy random variables [20-22], by considering the experts' ambiguous understanding of means and variances of random variables, a concept of random fuzzy variables was proposed, and mathematical programming problems with random fuzzy variables were formulated together with the development of a simulation-based approximate solution method [24].

Under these circumstances, as a first attempt to tackle decision making problems in hierarchical organizations under random fuzzy environments, assuming cooperative behavior of the decision makers, we have formulated random fuzzy two-level linear programming problems [25]. To deal with the formulated random fuzzy two-level linear programming problems, considering the probabilities that the decision makers' objective function values are smaller than or equal to target variables, we introduce fuzzy goals of the decision makers for the probabilities. Then we adopt fractile criteria [26] to optimize the target variables under the condition that the degrees of possibility with respect to the attained probabilities are greater than or equal to certain permissible levels. Interactive fuzzy programming to obtain a satisfactory solution for the decision maker at the upper level in consideration of the cooperative relation between decision makers is presented [25].

However, in real-world decision making situations, it is often found that decision variables in random fuzzy two-level linear programming problems are not continuous but rather discrete. From such a viewpoint, in this paper, we formulate random fuzzy two-level integer programming problems as natural extensions of random fuzzy two-level linear programming problems with continuous variables [25]. Through fractile criteria with possibility, by considering the cooperative relation between decision makers, we present interactive fuzzy programming for random fuzzy two-level integer programming problems. It is shown that all of the problems to be solved in the proposed interactive fuzzy programming become nonlinear integer programming problems and approximate optimal solutions can be obtained through the genetic algorithms with double strings for nonlinear integer programming. An illustrative numerical example is provided to demonstrate the feasibility and efficiency of the proposed method.

\section{Random Fuzzy Variables}

In the framework of stochastic programming, it is implicitly assumed that the uncertain parameter which well represents the stochastic factor of real systems can be definitely expressed as a single random variable. This means that the realized values of random parameters under the occurrence of some event are assumed to be definitely represented with real values.

Depending on the situations, however, it is natural to consider that the possible realized values of these random parameters are often only ambiguously known to the experts. In this case, it may be more appropriate to interpret the experts' ambiguous understanding of the realized values of random parameters as fuzzy numbers. From such a point of view, a fuzzy random variable was first introduced by Kwakernaak [20], and its mathematical basis was constructed by Puri and Ralescu [21]. An overview of the developments of fuzzy random variables was found in the recent article of Gil et al. [27].

From the expert's experimental point of view, however, the experts may think of a collection of random variables to be appropriate to express stochastic factors rather than only a single random variables. In this case, reflecting the expert's conviction degree that each of random variables properly represents the stochastic factor, it would be quite reasonable to assign the different degrees of possibility to each of random variables. For handling such an uncertain parameter, a random fuzzy variable was defined by Liu [24] as a function from a possibility space to a collection of random variables, which is considered to be an extended concept of fuzzy variable [28]. It should be noted here that the fuzzy variables can be viewed as another way of dealing with the imprecision which was originally represented by fuzzy sets. Although we can employ Liu's definition, for consistently discussing various concepts in relation to the fuzzy sets, we define the random fuzzy variables by extending not the fuzzy variables but the fuzzy sets.

Definition 1 (Random fuzzy variables) Let $\Gamma$ be a collection of random variables. Then, a random fuzzy variable $\overline{\tilde{C}}$ is defined by its membership function

$$
\mu_{\tilde{\tilde{C}}}: \Gamma \rightarrow[0,1] .
$$

In Definition 1, the membership function $\mu_{\overline{\tilde{C}}}$ assigns each random variable $\bar{\gamma} \in \Gamma$ to a real number $\mu_{\overline{\tilde{C}}}(\bar{\gamma})$. 
It should be noted here that if $\Gamma$ is defined as $\mathbb{R}$, then (1) becomes equivalent to the membership function of an ordinary fuzzy set. In this sense, a random fuzzy variable can be regarded as an extended concept of fuzzy sets. On the other hand, if $\Gamma$ is defined as a singleton $\Gamma=\{\bar{\gamma}\}$ and $\mu_{\overline{\tilde{C}}}(\bar{\gamma})=1$, then the corresponding random fuzzy variable $\overline{\tilde{C}}$ can be viewed as an ordinary random variable.

When taking account of the imprecise nature of the realized values of random variables, it would be appropriate to employ the concept of fuzzy random variables. However, it should be emphasized here that if mean and/ or variance of random variables are specified by the expert as a set of real values or fuzzy sets, such uncertain parameters can be represented by not fuzzy random variables but random fuzzy variables.

As a simple example of random fuzzy variables, we consider a Gaussian random variable whose mean value is not definitely specified as a constant. For example, when some random parameter $\bar{\gamma}$ is represented by the Gaussian random variable $N\left(s_{i}, 10^{2}\right)$ where the expert identifies a set $\left\{s_{1}, s_{2}, s_{3}\right\}$ of possible mean values as $\left(s_{1}, s_{2}, s_{3}\right)=(90,100,110)$, if the membership function $\mu_{\overline{\tilde{C}}}$ is defined by

$$
\mu_{\tilde{\tilde{C}}}(\bar{\gamma})= \begin{cases}0.5, & \text { if } \bar{\gamma} \sim N\left(90,10^{2}\right) \\ 0.7, & \text { if } \bar{\gamma} \sim N\left(100,10^{2}\right) \\ 0.3, & \text { if } \bar{\gamma} \sim N\left(90,10^{2}\right) \\ 0, & \text { otherwise }\end{cases}
$$

then $\overline{\tilde{C}}$ is a random fuzzy variable. More generally, when the mean values are expressed as fuzzy sets or fuzzy numbers, the corresponding random variable with the fuzzy mean is represented by a random fuzzy variable.

\section{Random Fuzzy Two-Level Integer Programming Problems}

As natural extensions of random fuzzy two-level linear programming problems with continuous variables [25], throughout of this paper, consider random fuzzy two-level integer programming problems. Realizing that the realworld decision making problems are often formulated as mathematical programming problems with integer decision variables, we consider the random fuzzy two-level integer programming problems formulated as

$$
\left.\begin{array}{ll}
\underset{\text { for DM1 }}{\operatorname{minimize}} & z_{1}\left(\boldsymbol{x}_{1}, \boldsymbol{x}_{2}\right)=\overline{\tilde{\boldsymbol{C}}}_{11} \boldsymbol{x}_{1}+\overline{\tilde{\boldsymbol{C}}}_{12} \boldsymbol{x}_{2} \\
\underset{\text { for DM2 }}{\operatorname{minimize}} & z_{2}\left(\boldsymbol{x}_{1}, \boldsymbol{x}_{2}\right)=\overline{\tilde{\boldsymbol{C}}}_{21} \boldsymbol{x}_{1}+\overline{\tilde{\boldsymbol{C}}}_{22} \boldsymbol{x}_{2} \\
\text { subject to } & A_{1} \boldsymbol{x}_{1}+A_{2} \boldsymbol{x}_{2} \leq \boldsymbol{b} \\
& x_{l j_{l}} \in\left\{0,1, \cdots, v_{l j_{l}}\right\}, j_{l}=1,2, \cdots, n_{l}, l=1,2 .
\end{array}\right\}
$$

where the two objective functions $z_{1}$ and $z_{2}$ are those of DM1 and DM2, respectively, and"minimize" and "minimize " mean that DM1 and DM2 areminimizers for their objective functions. Moreover, $\boldsymbol{x}_{1}$ is an $n_{1}$ dimensional integerdecision variable column vector for the decision maker at the upper level (DM1), $\boldsymbol{x}_{2}$ is an $n_{2}$ dimensional integer decision variable column vector for the decision maker at the lower level (DM2), $A_{j}, j=1,2$, are $m \times n_{j}$ coefficient matrices, $v_{l j_{l}}, j_{l}=1,2, \cdots, n_{l}, l=$ 1,2 , are positive integer values, and $\boldsymbol{b}$ is an $\mathrm{m}$ dimensional column vector.

Observing that the real data with uncertainty are often distributed normally, from the practical point of view, we assume that each of $\overline{\tilde{C}}_{l j k}, k=1,2, \cdots, n_{j}$ of $\overline{\tilde{C}}_{l j}, l=1,2$, $j=1,2$ is the Gaussian random variable with fuzzy mean value $\tilde{M}_{l j k}$ which is represented by an $L-R$ fuzzy number characterized by the membership function

$$
\mu_{\tilde{M}_{l j k}}(\tau)= \begin{cases}L\left(\frac{m_{l j k}-\tau}{\alpha_{l j k}}\right) & \text { if } m_{l j k} \geq \tau \\ R\left(\frac{\tau-m_{l j k}}{\beta_{l j k}}\right) & \text { if } m_{l j k}<\tau,\end{cases}
$$

where the shape functions $L$ and $R$ arenonincreasing continuous functions from $[0, \infty)$ to $[0,1], m_{l j k}$ is the mean value, and $\alpha_{l j k}$ and $\beta_{l j k}$ are positive numbers which represent left and right spreads. Figure 1 illustrates an example of the membership function $\mu_{\tilde{M}_{j k}}(\tau)$.

Let $\Gamma$ be a collection of all possible Gaussian random variables $N\left(\frac{s}{\tilde{C}}, \sigma^{2}\right)$ where $s \in(-\infty, \infty)$ and $\sigma^{2} \in$ $(0, \infty)$. Then, $\tilde{C}_{l j k}$ is expressed as a random fuzzy variable with the membership function

$$
\mu_{\overline{\tilde{C}}_{l j k}}\left(\bar{\gamma}_{l j k}\right)=\left\{\mu_{\overline{\tilde{M}}_{l j k}}\left(s_{l j k}\right) \mid \bar{\gamma}_{l j k} \sim N\left(s_{l j k}, \sigma_{l j k}^{2}\right)\right\}, \forall \bar{\gamma}_{l j k} \in \Gamma .
$$

Through the Zadeh's extension principle, in view of (4), the membership function of a random fuzzy variable corresponding to each of objective functions $z_{l}\left(\boldsymbol{x}_{1}, \boldsymbol{x}_{2}\right)$, $l=1,2$ is given as

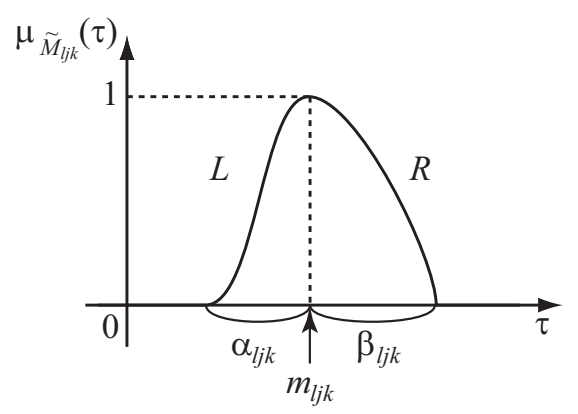

Figure 1. An example of the membership function $\mu_{\tilde{M}_{i j k}}(\tau)$. 


$$
\begin{aligned}
& \mu_{\overline{\tilde{c}}_{l} \boldsymbol{x}}\left(\bar{u}_{l}\right)=\sup _{\bar{\gamma}_{l}}\left\{\min _{1 \leq k \leq n_{j}, j=1,2} \mu_{\overline{\tilde{c}}_{l j k}}\left(\bar{\gamma}_{l j k}\right) \mid \bar{u}_{l}=\sum_{j=1}^{2} \sum_{k=1}^{n_{j}} \bar{\gamma}_{l j k} x_{j k}\right\} \\
& =\sup _{s_{l}}\left\{\min _{1 \leq k \leq n_{j}, j=1,2} \mu_{\tilde{M}_{l j k}}\left(s_{l j k}\right) \mid \bar{u}_{l} \sim N\left(\sum_{j=1}^{2} \sum_{k=1}^{n_{j}} s_{l j k} x_{j k}, V_{l}(\boldsymbol{x})\right)\right\}
\end{aligned}
$$

where $\bar{\gamma}_{l}=\left(\bar{\gamma}_{l 11}, \cdots, \bar{\gamma}_{l 1 n_{1}}, \bar{\gamma}_{l 21}, \cdots, \bar{\gamma}_{l 2 n_{2}}\right)$,

$s_{l}=\left(s_{l 11}, \cdots, s_{l 1 n_{1}}, s_{l 21}, \cdots, s_{l 2 n_{2}}\right)$, and

$$
V_{l}(\boldsymbol{x})=\sum_{j=1}^{2} \sum_{k=1}^{n_{j}} \sigma_{l j k}^{2} x_{j k}^{2} \text {. }
$$

\section{Fractile Criteria with Possibility Incorporating Fuzzy Goals}

Assuming that the decision makers (DMs) concerns about the probabilities that their own objective function values $\tilde{\boldsymbol{C}}_{l} \boldsymbol{x}$ are smaller than or equal to certain target values $f_{l}, l=1,2$, we introduce the probabilities

$P\left(\omega \mid \tilde{C}_{l}(\omega) \boldsymbol{x} \leq f_{l}\right)$ which are expressed as fuzzy sets $\tilde{P}_{l}$ with the membership functions

$$
\mu_{\tilde{P}_{l}}\left(p_{l}\right)=\sup _{\tilde{u}_{l}}\left\{\mu_{\tilde{\tilde{c}}_{l} x}\left(\tilde{u}_{l}\right) \mid p_{l}=P\left(\omega \mid \tilde{u}_{l}(\omega) \leq f_{l}\right)\right\},
$$

where $f_{l}, l=1,2$ are the initial target values specified by the DMs as constants.

Considering the imprecise nature of the DMs' judgments for the probabilities $\tilde{P}_{l-}$ with respect to the random fuzzy objective values $\overline{\tilde{\boldsymbol{C}}}_{l} \boldsymbol{x}, l=1,2$, we introduce the fuzzy goals $\tilde{G}_{l}, l=1,2$ such as " $\tilde{P}_{l}$ should be greater than or equal to a certain value." Such fuzzy goals $\tilde{G}_{l}, l=1,2$ can be quantified by eliciting corresponding membership functions

$$
\mu_{\tilde{G}_{l}}(p)= \begin{cases}0 & \text { if } p \leq p_{l}^{0} \\ g_{l}(p) & \text { if } p_{l}^{0} \leq p \leq p_{l}^{1}, l=1,2 . \\ 1 & \text { if } p_{l}^{1} \leq p\end{cases}
$$

where $g_{l}(p), l=1,2$ are nondecreasing functions. Figure 2 illustrates a possible shape of the membership function for the fuzzy goal $\tilde{G}_{l}$.

Recalling that the membership function is regarded as a possibility distribution, the degree of possibility that the probability $\tilde{P}_{l}$ attains the fuzzy goal $\tilde{G}_{l}$ is expressed as

$$
\Pi_{\tilde{P}_{l}}\left(\tilde{G}_{l}\right)=\sup _{p_{l}} \min \left\{\mu_{\tilde{P}_{l}}\left(p_{l}\right), \mu_{\tilde{G}_{l}}\left(p_{l}\right)\right\}, l=1,2 .
$$

Figure 3 illustrates the degree of possibility $\Pi_{\tilde{P}_{l}}\left(\tilde{G}_{l}\right)$. Now, assuming that the DMs are willing to maximize the degrees of possibility with respect to the attained probability, we consider the possibility-based probability model for random fuzzy two-level programming problems formulated as

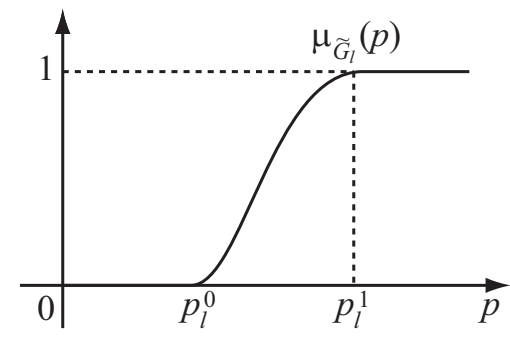

Figure 2. An example of a membership function $\mu_{\widehat{G}_{t}}(p)$ of a fuzzy goal $\tilde{G}_{\boldsymbol{l}}$.

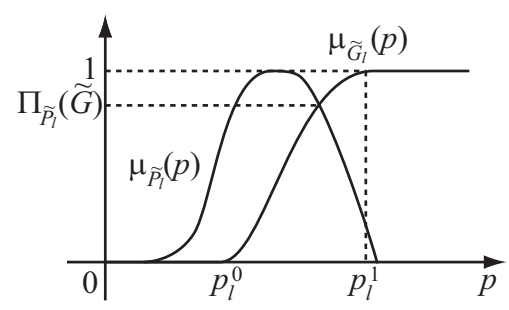

Figure 3. The degree of possibility $\Pi_{\tilde{P}_{l}}\left(\tilde{G}_{t}\right)$.

$$
\left.\begin{array}{ll}
\underset{\text { for DM1 }}{\operatorname{maximize}} & \Pi_{\tilde{P}_{1}}\left(\tilde{G}_{1}\right) \\
\underset{\text { for DM2 }}{\operatorname{maximize}} & \Pi_{\tilde{P}_{2}}\left(\tilde{G}_{2}\right) \\
\text { subject to } & A_{1} \boldsymbol{x}_{1}+A_{2} \boldsymbol{x}_{2} \leq \boldsymbol{b} \\
& x_{l j_{l}} \in\left\{0,1, \cdots, v_{l j_{l}}\right\}, j_{l}=1,2, \cdots, n_{l}, l=1,2
\end{array}\right\}
$$

or equivalently

$$
\left.\begin{array}{cl}
\underset{\text { for DM1 }}{\operatorname{maximize}} & \mu_{1}\left(f_{1}\right) \\
\underset{\text { for DM2 }}{\operatorname{maximize}} & \mu_{2}\left(f_{2}\right) \\
\text { subject to } & \Pi_{\tilde{P}_{1}}\left(\tilde{G}_{1}\right) \geq h_{1} \\
& \Pi_{\tilde{P}_{2}}\left(\tilde{G}_{2}\right) \geq h_{2} \\
& A_{1} \boldsymbol{x}_{1}+A_{2} \boldsymbol{x}_{2} \leq \boldsymbol{b} \\
& x_{l j_{l}} \in\left\{0,1, \cdots, v_{l j_{l}}\right\}, j_{l}=1,2, \cdots, n_{l}, l=1,2,
\end{array}\right\}
$$

where $h_{1}$ and $h_{2}$ are permissible possibility levels specified by the DMs, and $\mu_{1}$ and $\mu_{2}$ are the membership functions of the fuzzy goals for the target variables $f_{1}$ and $f_{2}$, respectively.

It should be noted here that the bilevel programming problem (10) involves the possibility constraints $\Pi_{\tilde{P}_{l}}\left(\tilde{G}_{l}\right) \geq h_{l}, l=1,2$. Fortunately, however, the following theorem holds for the constraints $\Pi_{\tilde{P}_{l}}\left(\tilde{G}_{l}\right) \geq h_{l}, l=1,2$ in (10).

Theorem 1. Let $\Phi$ denote a probability distribution function of the standard Gaussian random variable $N(0$, 1). Then, $\Pi_{\tilde{P}_{l}}\left(\tilde{G}_{l}\right) \geq h_{l}, l=1,2$ in (10) is equivalently transformed into

$$
\sum_{j=1}^{2} \sum_{k=1}^{n_{j}}\left\{m_{l j k}-L^{\star}\left(h_{l}\right) \alpha_{l j k}\right\} x_{j k}+\Phi^{-1}\left(\mu_{\tilde{G}_{l}}^{\star}\left(h_{l}\right)\right) \sqrt{V_{l}(x)} \leq f_{l},
$$


where $L^{\star}\left(h_{l}\right)$ is a pseudo inverse functions defined as $L^{\star}\left(h_{l}\right)=\sup \left\{t \mid L(t)>h_{l}, r \geq 0\right\}$ and $\Phi^{-1}$ is the inverse function of $\Phi$.

Proof

From (8), the constraints $\Pi_{\tilde{P}_{l}}\left(\tilde{G}_{l}\right) \geq h_{l}, l=1,2$ in (10) is equivalently replaced by the condition that there exists a $p$ such that $\mu_{\tilde{P}_{l}}\left(p_{l}\right) \geq h_{l}$ and $\mu_{\tilde{G}_{l}}\left(p_{l}\right) \geq h_{l}$, namely,

$$
\begin{aligned}
& \sup _{\bar{s}_{l}} \min _{1 \leq k \leq n_{j}, j=1,2}\left\{\mu_{\tilde{M}_{l j k}}\left(s_{l j k}\right) \mid p_{l}=P\left(\omega \mid \bar{u}_{l}(\omega) \leq f_{l}\right),\right. \\
& \left.\bar{u}_{l} \sim N\left(\sum_{j=1}^{2} \sum_{k=1}^{n_{j}} s_{l j k} x_{j k}, V_{l}(\boldsymbol{x})\right)\right\} \geq h_{l}
\end{aligned}
$$

and $p_{l} \geq \mu_{\tilde{G}_{l}}^{\star}\left(h_{l}\right), l=1,2$, where $\mu_{\tilde{G}_{l}}^{\star}\left(h_{l}\right)$ are pseudo inverse functions defined as

$\mu_{\tilde{G}_{l}}^{\star}\left(h_{l}\right)=\inf \left\{p_{l} \mid \mu_{\tilde{G}_{l}}\left(p_{l}\right) \geq h_{l}\right\}, l=1,2$. This implies that there exists a vector $\left(p_{l}, s_{l}, \bar{u}_{l}\right), l=1,2$ such that

$$
\begin{gathered}
\min _{1 \leq k \leq n_{j}, j=1,2} \mu_{\tilde{M}_{l j k}}\left(s_{l j k}\right) \geq h_{l}, \bar{u}_{l} \sim N\left(\sum_{j=1}^{2} \sum_{k=1}^{n_{j}} s_{l j k} x_{j k}, V_{l}(\boldsymbol{x})\right), \\
p_{l}=P\left(\omega \mid \bar{u}_{l}(\omega) \leq f_{l}\right), p_{l} \geq \mu_{\tilde{G}_{l}}^{\star},
\end{gathered}
$$

whichcan be equivalently transformed into the condition that there exists a vector $\left(s_{l}, \bar{u}_{l}\right)$ such that

$$
\begin{gathered}
\mu_{\tilde{M}_{l j k}}\left(s_{l j k}\right) \geq h_{l}, \bar{u}_{l} \sim N\left(\sum_{j=1}^{2} \sum_{k=1}^{n_{j}} s_{l j k} x_{j k}, V_{l}(\boldsymbol{x})\right), \\
P\left(\omega \mid \bar{u}_{l}(\omega) \leq f_{l}\right) \geq \mu_{\tilde{G}_{l}}^{\star}, l=1,2, j=1,2, k=1, \cdots, n_{j} .
\end{gathered}
$$

In view of (3), it follows that

$$
\begin{gathered}
\mu_{\tilde{M}_{l j k}}\left(s_{l j k}\right) \geq h_{l} \\
\Leftrightarrow s_{l j k} \in\left[m_{l j k}-L^{\star}\left(h_{l}\right) \alpha_{l j k}, m_{l j k}-R^{\star}\left(h_{l}\right) \beta_{l j k}\right],
\end{gathered}
$$

where $L^{\star}\left(h_{l}\right)$ and $R^{\star}\left(h_{l}\right)$ are pseudo inverse functions defined as $L^{\star}\left(h_{l}\right)=\sup \left\{t \mid L(t) \geq h_{l}\right\}$ and $R^{\star}\left(h_{l}\right)=\sup \left\{t \mid L(t) \geq h_{l}\right\}$. Hence, (12) is rewritten as theequivalent condition that there exists a $\bar{u}_{l}$ such that

$$
P\left(\omega \mid \bar{u}_{l}(\omega) \leq f_{l}\right), p_{l} \geq \mu_{\tilde{G}_{l}}^{\star},
$$

$$
\bar{u}_{l} \sim N\left(\sum_{j=1}^{2} \sum_{k=1}^{n_{j}}\left\{m_{l j k}-L^{\star}\left(h_{l}\right) \alpha_{l j k}\right\} x_{j k}, V_{l}(\boldsymbol{x})\right),
$$

Since $P\left(\omega \mid \bar{u}_{l}(\omega) \leq f_{l}\right)$ is transformed into

$$
\begin{aligned}
& P\left(\omega \mid \frac{\bar{u}_{l}-\sum_{j=1}^{2} \sum_{k=1}^{n_{j}}\left\{m_{l j k}-L^{\star}\left(h_{l}\right) \alpha_{l j k}\right\} x_{j k}}{\sqrt{V_{l}(\boldsymbol{x})}}\right. \\
& \left.\leq \frac{f_{l}-\sum_{j=1}^{2} \sum_{k=1}^{n_{j}}\left\{m_{l j k}-L^{\star}\left(h_{l}\right) \alpha_{l j k}\right\} x_{j k}}{\sqrt{V_{l}(\boldsymbol{x})}}\right)
\end{aligned}
$$

in consideration of

$$
\frac{\bar{u}_{l}-\sum_{j=1}^{2} \sum_{k=1}^{n_{j}}\left\{m_{l j k}-L^{\star}\left(h_{l}\right) \alpha_{l j k}\right\} x_{j k}}{\sqrt{V_{l}(\boldsymbol{x})}} \sim N(0,1),
$$

(13) isequivalently transformed as

$$
\Phi\left(\frac{f_{l}-\sum_{j=1}^{2} \sum_{k=1}^{n_{j}}\left\{m_{l j k}-L^{\star}\left(h_{l}\right) \alpha_{l j k}\right\} x_{j k}}{\sqrt{V_{l}(\boldsymbol{x})}}\right) \geq \mu_{\tilde{G}_{l}}^{\star},
$$

where $\Phi$ is a probability distribution function of the standard Gaussian randomvariable $N(0,1)$.

From the monotone increasingnessof $\Phi$, (14) is rewritten as

$$
\sum_{j=1}^{2} \sum_{k=1}^{n_{j}}\left\{m_{l j k}-L^{\star}\left(h_{l}\right) \alpha_{l j k}\right\} x_{j k}+\Phi^{-1}\left(\mu_{\tilde{G}_{l}}^{\star}\left(h_{l}\right)\right) \sqrt{V_{l}(\boldsymbol{x})} \leq f_{l}
$$

where $\Phi^{-1}$ is the inverse function of $\Phi$.

From (11)-(15), it holds that

$$
\begin{aligned}
& \Pi_{\tilde{P}_{l}}\left(\tilde{G}_{l}\right) \geq h_{l} \\
& \Leftrightarrow \sum_{j=1}^{2} \sum_{k=1}^{n_{j}}\left\{m_{l j k}-L^{\star}\left(h_{l}\right) \alpha_{l j k}\right\} x_{j k}+\Phi^{-1}\left(\mu_{\tilde{G}_{l}}^{\star}\left(h_{l}\right)\right) \sqrt{V_{l}(\boldsymbol{x})} \leq f_{l} .
\end{aligned}
$$

This completes the proof of the theorem.

Due to Theorem 1, the two-level integer programming problem with the possibility constraints (9) is equivalently transformed into (17) or equivalently (18)

$$
\begin{aligned}
& \underset{\text { for DM1 }}{\operatorname{maximize}} \quad \mu_{1}\left(f_{1}\right) \\
& \underset{\text { for DM2 }}{\operatorname{maximize}} \quad \mu_{2}\left(f_{2}\right) \\
& \text { subject to } \quad \sum_{j=1}^{2} \sum_{k=1}^{n_{j}}\left\{m_{1 j k}-L^{\star}\left(h_{1}\right) \alpha_{1 j k}\right\} x_{1 j k}+\Phi^{-1}\left(\mu_{\tilde{G}_{1}}^{\star}\left(h_{1}\right)\right) \sqrt{V_{1}(\boldsymbol{x})} \leq f_{1} \\
& \sum_{j=1}^{2} \sum_{k=1}^{n_{j}}\left\{m_{2 j k}-L^{\star}\left(h_{2}\right) \alpha_{2 j k}\right\} x_{2 j k}+\Phi^{-1}\left(\mu_{\tilde{G}_{2}}^{\star}\left(h_{2}\right)\right) \sqrt{V_{2}(\boldsymbol{x})} \leq f_{2} \\
& A_{1} \boldsymbol{x}_{1}+A_{2} \boldsymbol{x}_{2} \leq \boldsymbol{b} \\
& x_{l j_{l}} \in\left\{0,1, \cdots, v_{l j_{l}}\right\}, j_{l}=1,2, \cdots, n_{l}, l=1,2 \text {, }
\end{aligned}
$$




$$
\left.\begin{array}{ll}
\underset{\text { for DM1 }}{\operatorname{maximize}} & \mu_{1}\left(Z_{1}^{\Pi, F}\left(\boldsymbol{x}_{1}, \boldsymbol{x}_{2}\right)\right) \\
\underset{\text { for DMze }}{\operatorname{maximize}} & \mu_{2}\left(Z_{2}^{\Pi, F}\left(\boldsymbol{x}_{1}, \boldsymbol{x}_{2}\right)\right) \\
\text { subject to } & A_{1} \boldsymbol{x}_{1}+A_{2} \boldsymbol{x}_{2} \leq \boldsymbol{b} \\
& x_{l_{l}} \in\left\{0,1, \cdots, v_{l_{l}}\right\}, j_{l}=1,2, \cdots, n_{l}, l=1,2,
\end{array}\right\}
$$

where

$$
\begin{aligned}
Z_{l}^{\Pi, F}\left(\boldsymbol{x}_{1}, \boldsymbol{x}_{2}\right)= & \sum_{j=1}^{2} \sum_{k=1}^{n_{j}}\left\{m_{l j k}-L^{\star}\left(h_{l}\right) \alpha_{l j k}\right\} x_{j k} \\
& +\Phi^{-1}\left(\mu_{\tilde{G}_{l}}^{\star}\left(h_{l}\right)\right) \sqrt{V_{l}(\boldsymbol{x})}, l=1,2 .
\end{aligned}
$$

It should be emphasized here that (18) is a deterministic two-level nonlinear integer programming problem.

\section{Interactive Fuzzy Programming}

In order to obtain an initial candidate for an overall satisfactory solution to (9) or (17), it would be useful for DM1 to find a solution which maximize the smallerdegree of satisfaction between the two DMs by solving the maximin problem

$$
\left.\begin{array}{ll}
\operatorname{maximize} & \min \left\{\mu_{1}\left(Z_{1}^{\Pi, F}\left(\boldsymbol{x}_{1}, \boldsymbol{x}_{2}\right)\right), \mu_{2}\left(Z_{2}^{\Pi, F}\left(\boldsymbol{x}_{1}, \boldsymbol{x}_{2}\right)\right)\right\} \\
\text { subject to } & A_{1} \boldsymbol{x}_{1}+A_{2} \boldsymbol{x}_{2} \leq \boldsymbol{b} \\
& x_{l_{j_{l}}} \in\left\{0,1, \cdots, v_{l_{l}}\right\}, j_{l}=1,2, \cdots, n_{l}, l=1,2,
\end{array}\right\}
$$

By introducing an auxiliary variable $v$, this problem is written as

$$
\left.\begin{array}{ll}
\operatorname{maximize} & v \\
\text { subject to } & \mu_{1}\left(Z_{1}^{\Pi, F}\left(\boldsymbol{x}_{1}, \boldsymbol{x}_{2}\right)\right) \geq v \\
& \mu_{2}\left(Z_{2}^{\Pi, F}\left(\boldsymbol{x}_{1}, \boldsymbol{x}_{2}\right)\right) \geq v \\
& A_{1} \boldsymbol{x}_{1}+A_{2} \boldsymbol{x}_{2} \leq \boldsymbol{b} \\
& x_{l_{j l}} \in\left\{0,1, \cdots, v_{l_{l}}\right\}, j_{l}=1,2, \cdots, n_{l}, l=1,2,
\end{array}\right\}
$$

Although the membership function does not always need to be linear, for the sake of simplicity, we adopt a linear membership function which characterizes the fuzzy goal of each decision maker. The linear membership functions $\mu_{l}, l=1,2$ are defined as

$$
\begin{aligned}
& \mu_{l}\left(Z_{l}^{\Pi, F}\left(\boldsymbol{x}_{1}, \boldsymbol{x}_{2}\right)\right) \\
= & \begin{cases}1 & \text { if } Z_{l}^{\Pi, F}\left(\boldsymbol{x}_{1}, \boldsymbol{x}_{2}\right) \leq z_{l}^{1} \\
\frac{Z_{l}^{\Pi, F}\left(\boldsymbol{x}_{1}, \boldsymbol{x}_{2}\right)-z_{l}^{0}}{z_{l}^{1}-z_{l}^{0}} & \text { if } z_{l}^{1}<Z_{l}^{\Pi, F}\left(\boldsymbol{x}_{1}, \boldsymbol{x}_{2}\right)<z_{l}^{0} \\
0 & \text { if } Z_{l}^{\Pi, F}\left(\boldsymbol{x}_{1}, \boldsymbol{x}_{2}\right) \geq z_{l}^{0} .\end{cases}
\end{aligned}
$$

Then, (21) is equivalently transformed as (23)

If DM1 is satisfied with the membership function values $\mu_{l}\left(Z_{l}^{\Pi, F}\left(\boldsymbol{x}_{1}^{*}, \boldsymbol{x}_{2}^{*}\right)\right), l=1,2$, the corresponding opti mal solution $\boldsymbol{x}^{*}$ to (21) is regarded as the satisfactorysolution. Otherwise, by introducing the constraint that $\mu_{1}\left(Z_{1}^{\Pi, F}(\boldsymbol{x})\right)$ is larger than or equal to the minimal satisfactory level $\delta \in(0,1)$ specified by DM1, we consider the problem of maximizing the membership function $\mu_{2}\left(Z_{2}^{\Pi, F}\left(\boldsymbol{x}_{1}, \boldsymbol{x}_{2}\right)\right)$ formulated as

$$
\left.\begin{array}{ll}
\operatorname{maximize} & \mu_{2}\left(Z_{2}^{\Pi, F}\left(\boldsymbol{x}_{1}, \boldsymbol{x}_{2}\right)\right) \\
\text { subject to } & \mu_{1}\left(Z_{1}^{\Pi, F}\left(\boldsymbol{x}_{1}, \boldsymbol{x}_{2}\right)\right) \geq \delta \\
& A_{1} \boldsymbol{x}_{1}+A_{2} \boldsymbol{x}_{2} \leq \boldsymbol{b} \\
& x_{l j_{l}} \in\left\{0,1, \cdots, v_{l j_{l}}\right\}, j_{l}=1,2, \cdots, n_{l}, l=1,2,
\end{array}\right\}
$$

or equivalently (25)

In general, when the objective functions of DM1 and DM2 conflict with eachother, it should be noted here that the larger the minimal satisfactory level $\delta$ for $\mu_{1}$ is specified by DM1, the smaller the satisfactory degree for $\mu_{2}$ becomes, whichmay lead to the improper satisfac-

$$
\left.\begin{array}{cl}
\text { maximize } & v \\
\text { subject to } & \sum_{j=1}^{v} \sum_{k=1}^{n_{j}}\left\{m_{1 j k}-L^{\star}\left(h_{1}\right) \alpha_{1 j k}\right\} x_{j k}+\Phi^{-1}\left(\mu_{\tilde{G}_{1}}^{\star}\left(h_{1}\right)\right) \sqrt{V_{1}(\boldsymbol{x})} \leq\left(z_{1}^{1}-z_{1}^{0}\right) v+z_{1}^{0} \\
& \left.\sum_{j=1}^{2} \sum_{k=1}^{n_{j}}\left\{m_{2 j k}-L^{\star}\left(h_{2}\right) \alpha_{2 j k}\right\} x_{j k}+\Phi^{-1}\left(\mu_{\tilde{G}_{2}}^{\star}\left(h_{2}\right)\right) \sqrt{V_{2}(\boldsymbol{x})} \leq\left(z_{2}^{1}-z_{2}^{0}\right) v+z_{2}^{0}\right\} \\
& A_{1} \boldsymbol{x}_{1}+A_{2} \boldsymbol{x}_{2} \leq \boldsymbol{b} \\
& x_{l_{j l}} \in\left\{0,1, \cdots, v_{l_{l}}\right\}, j_{l}=1,2, \cdots, n_{l}, l=1,2, \\
\text { maximize } & \sum_{j=1}^{2} \sum_{k=1}^{n_{j}}\left\{m_{2 j k}-L^{\star}\left(h_{2}\right) \alpha_{2 j k}\right\} x_{j k}+\Phi^{-1}\left(\mu_{\tilde{G}_{2}}^{\star}\left(h_{2}\right)\right) \sqrt{V_{2}(\boldsymbol{x})} \\
\text { subject to } & \left.\sum_{j=1}^{2} \sum_{k=1}^{n_{j}}\left\{m_{1 j k}-L^{\star}\left(h_{1}\right) \alpha_{1 j k}\right\} x_{j k}+\Phi^{-1}\left(\mu_{\tilde{G}_{1}}^{\star}\left(h_{1}\right)\right) \sqrt{V_{1}(\boldsymbol{x})} \leq\left(z_{1}^{1}-z_{1}^{0}\right) \delta+z_{1}^{0}\right\} \\
& A_{1} \boldsymbol{x}_{1}+A_{2} \boldsymbol{x}_{2} \leq \boldsymbol{b} \\
& x_{l j_{l}} \in\left\{0,1, \cdots, v_{l j l}\right\}, j_{l}=1,2, \cdots, n_{l}, l=1,2,
\end{array}\right\}
$$


tory balance between DM1 and DM2 due to the large difference between the membership function values of both DMs.

In order to derive the satisfactory solution which has well-balanced membership function values between both DMs, by introducing the ratio $\Delta$ expressed as

$$
\Delta=\frac{\mu_{2}\left(Z_{2}^{\Pi, F}\left(\boldsymbol{x}_{1}, \boldsymbol{x}_{2}\right)\right)}{\mu_{1}\left(Z_{1}^{\Pi, F}\left(\boldsymbol{x}_{1}, \boldsymbol{x}_{2}\right)\right)},
$$

the lower bound $\Delta_{\min }$ and the upper bound of $\Delta_{\max }$ of $\Delta$, specified by DM1, are introduced to determine whether or not the ratio $\Delta$ is appropriate. To be more explicit, if it holds that

$$
\Delta \in\left[\Delta_{\min }, \Delta_{\max }\right]
$$

then DM1 regards the corresponding solution as a preferable candidate for the satisfactory solution with wellbalanced membership function values.

Now we summarize a procedure of interactive fuzzy programming through fractile criteria with possibility in order to derive a satisfactory solution.

\section{Interactive Fuzzy Programming through Fractile Criteria with Possibility}

Step 0: Ask DMs to specify the initial target values $f_{l}, l=1,2$, and determine the membership functions $\mu_{\tilde{G}_{l}}, l=1,2$.

Step 1: Ask DM1 to specify the permissible possibility levels $h_{l}, l=1,2$.

Step 2: Ask DMs to determine the membership functions $\mu_{l}, l=1,2$.

Step 3: For the current $h_{l}, l=1,2$, solve the maxmin problems (20).

Step 4: DM1 is supplied with the current values of the membership functions $\mu_{1}$ and $\mu_{2}$ for the optimal solution obtained in step 3. If DM1 is satisfied with the current membership function values, then stop. If DM1 is not satisfied and prefers to update $h_{l}, l=1,2$, ask DM1 to update $h_{l}$, and return to step 3. Otherwise, ask DM1 to specify the minimal satisfactory level $\delta$ for $\mu_{1}\left(Z_{1}^{\Pi, F}(\boldsymbol{x})\right)$ and the permissible range $\left[\Delta_{\min }, \Delta_{\max }\right]$ of the ratio $\Delta$.

Step 5: For the current minimal satisfactory level $\delta$, solve the membership function maximization problem (25).

Step 6: DM1 is supplied with the current values of the membership function $\mu_{1}, \mu_{2}$ and the ratio $\Delta$. If $\Delta \in\left[\Delta_{\min }, \Delta_{\max }\right]$ and DM1 is satisfied with the current membership function values, then stop. Otherwise, ask DM1 to update the minimal satisfactory level $\delta$, and return to step 5 .

In the proposed interactive fuzzy programming method, it is required to solve the nonlinear integer programming problems (20) and (25), which is apparently difficult to solve compared to linear integer programming problems and 0 -1nonlinear programming problems. In order to solve such difficult problems, in the following section, we introduce genetic algorithms designed for nonlinear integer programming problems.

\section{Genetic Algorithms for Nonlinear Integer Programming}

For solving linear integer programming problems on the framework of geneticalgorithms, Sakawa proposed GADSLPRRSU [29]. GADSLPRRSU is an abbreviation for genetic algorithms with double strings based on linear programming relaxation and reference solution updating. This method includes three key ideas: double strings (DS), linear programming relaxation (LPR), and reference solution updating (RSU). Unfortunately, however, due to nonlinearity, we cannot directly apply GADSLPRRSU for solving (20) and (25). However, we can introduce the revised GADSLPRRSU where GENOCOPIII [30,31] is employed for solving a nonlinear continuous relaxation problem.

As an efficient approximate solution method, the revised GADSLPRRSU are designed for nonlinear integer programming problems formulated as:

$$
\left.\begin{array}{cl}
\text { minimize } & f(\boldsymbol{x}) \\
\text { subject to } & g_{i}(\boldsymbol{x}) \leq 0, i=1,2, \ldots, m \\
& x_{j} \in\left\{0,1, \cdots, v_{j}\right\}, j=1,2, \cdots, n
\end{array}\right\}
$$

where $\boldsymbol{x}$ is an $n$ dimensional integer decision variable column vector. Furthermore, $f(\cdot)$ and

$g_{i}(\cdot), i=1,2, \cdots, m$ may be nonlinear.

Quite similar to genetic algorithms with double (GADS) [29], an individual is represented by a double string shown in Figure 4. In Figure 4, for a certain $j, s(j) \in\{1,2, \cdots, n\}$ represents an index of decision variable $x_{s(j)}$ in the solution space, while $y_{s(j)}, j=1,2, \cdots, n$ does the integer value among $\left\{0,1, \cdots, v_{j}\right\}$ of the $s(j)$ th decision variable $x_{s(j)}$.

Now we can summarize the computational procedures of the revised GADSLPRRSU as follows.

\section{Computational Procedures of the Revised GADSLPRRSU}

Step 0: Determine values of the parameters used in the genetic algorithm. Set the generation counter $t$ at 0 .

Step 1: Generate the initial population consisting of

\begin{tabular}{|c|c|c|c|}
\hline$s(1)$ & $s(2)$ & $\cdots$ & $s(n)$ \\
\hline$y_{s}(1)$ & $y_{s}(2)$ & $\cdots$ & $y_{s}(n)$ \\
\hline
\end{tabular}

Figure 4. Double string. 
$N$ individuals based on the information of the optimal solution to the continuous relaxation problem.

Step 2: Decode each individual in the current population and calculate its fitness based on the corresponding solution.

Step 3: If the termination condition is fulfilled, stop. Otherwise, let $t:=t+1$.

Step 4: Apply reproduction operator using elitist expected value selection after linear scaling.

Step 5: Apply crossover operator, called PMX (Partially Matched Crossover) for double string.

Step 6: Apply mutation based on the information of a solution to the continuous relaxation problem.

Step 7: Apply inversion operator, return to Step 2.

Further details of GADSLPRRSU and the revised GADSLPRRSU can be found in $[17,29,32]$.

\section{Numerical Example}

To demonstrate the feasibility and efficiency of the proposed method, consider the following two-level integer programming problem involving random fuzzy variable coefficients:

$$
\left.\begin{array}{cl}
\underset{f o r}{\operatorname{minimize}} & z_{1}\left(\boldsymbol{x}_{1}, \boldsymbol{x}_{2}\right)=\overline{\tilde{\boldsymbol{C}}}_{11} \boldsymbol{x}_{1}+\overline{\tilde{\boldsymbol{C}}}_{12} \boldsymbol{x}_{2} \\
\underset{\text { for DM2 }}{\operatorname{minimize}} & z_{2}\left(\boldsymbol{x}_{1}, \boldsymbol{x}_{2}\right)=\overline{\tilde{\boldsymbol{C}}}_{21} \boldsymbol{x}_{1}+\overline{\tilde{\boldsymbol{C}}}_{22} \boldsymbol{x}_{2} \\
\text { subject to } & a_{11} \boldsymbol{x}_{1}+a_{12} \boldsymbol{x}_{2} \leq b_{1} \\
& a_{21} \boldsymbol{x}_{1}+a_{22} \boldsymbol{x}_{2} \leq b_{2} \\
& a_{31} \boldsymbol{x}_{1}+a_{32} \boldsymbol{x}_{2} \leq b_{3} \\
& a_{41} \boldsymbol{x}_{1}+a_{42} \boldsymbol{x}_{2} \leq b_{4} \\
& a_{51} \boldsymbol{x}_{1}+a_{52} \boldsymbol{x}_{2} \leq b_{5} \\
& x_{l j_{2}} \in\{0,1, \cdots, 30\}, j_{2}=1,2,3, l=1,2 .
\end{array}\right\}
$$

Table 1 shows values of coefficients of constraints $a_{i}, i=1,2,3,4,5$ and $b_{i}, i=1,2,3,4,5$ and Table 2 shows

Table 1. Values of coefficients in constraints.

\begin{tabular}{cccccccc}
\hline & $a_{l 11}$ & $a_{l 12}$ & $a_{l 13}$ & $a_{l 21}$ & $a_{l 22}$ & $a_{l 23}$ & $\boldsymbol{b}$ \\
\hline$a_{1}$ & 15.00 & 20.00 & 20.00 & 0.00 & 0.00 & 0.00 & 1400 \\
$a_{2}$ & 0.00 & 0.00 & 0.00 & 2.50 & 9.00 & 4.00 & 430 \\
$a_{3}$ & 5.00 & 2.00 & 1.00 & 5.00 & 5.00 & 8.00 & 400 \\
$a_{4}$ & 3.00 & 8.00 & 4.00 & 7.00 & 8.00 & 8.00 & 600 \\
$a_{5}$ & 3.50 & 4.00 & 4.00 & 5.50 & 4.00 & 1.00 & 300 \\
\hline
\end{tabular}

Table 2. Values of $m_{l j k}, \alpha_{l j k}$ and $\sigma_{l j k}^{2}$.

\begin{tabular}{ccccccc}
\hline & $\tilde{c}_{l 11}$ & $\tilde{c}_{l 12}$ & $\tilde{c}_{l 13}$ & $\tilde{c}_{l 21}$ & $\tilde{c}_{l 22}$ & $\tilde{c}_{l 23}$ \\
\hline$m_{1 j k}$ & -7.00 & -4.00 & -5.00 & -3.00 & -5.00 & -3.00 \\
$m_{2 j k}$ & -4.00 & -3.00 & -4.00 & -2.00 & -4.00 & -3.00 \\
$a_{1 j k}$ & 0.70 & 0.50 & 0.80 & 0.40 & 0.70 & 0.60 \\
$a_{2 j k}$ & 0.90 & 0.80 & 0.70 & 0.40 & 0.60 & 0.50 \\
$\sigma_{1 j k}^{2}$ & 1.40 & 1.00 & 1.10 & 1.20 & 1.10 & 0.90 \\
$\sigma_{2 j k}^{2}$ & 1.00 & 1.00 & 1.20 & 1.20 & 0.80 & 1.00 \\
\hline
\end{tabular}

values of parameters of random fuzzy variables $m_{l j k}$, $\alpha_{l j k}$ and $\sigma_{l j k}^{2}, l=1,2, j=1,2, k=1,2, \cdots, 6$, where traingular fuzzy numbers are assumed for $\mu_{\tilde{M}_{l j k}}(\tau)$.

Through the use of this numerical example, it is now appropriate to illustrate the proposed interactive fuzzy programming.

For illustrative purposes, assume that DMs specify the initial target values as $f_{1}=-120.00$ and $f_{2}=90.00$ and determine the membership functions (7) for the probabilities $\tilde{P}_{1}$ and $\tilde{P}_{2}$ as linear ones by assessing $p_{1}^{0}=0.50, p_{2}^{0}=0.40, p_{1}^{1}=0.85, p_{2}^{1}=0.80$ and

$$
g_{1}(p)=\frac{p-0.5}{0.35}, g_{2}(p)=\frac{p-0.4}{0.4} \text {. }
$$

Also assume that DM1 specify the permissible possibility levels as $h_{1}=0.7$ and $h_{2}=0.7$. Furthermore, assume that the fuzzy goals for the target variables $f_{1}$ and $f_{2}$ are determined by the linear membership functions

$$
\mu_{l}\left(f_{l}\right)= \begin{cases}1 & \text { if } f_{l} \leq f_{l}^{1} \\ \frac{f_{l}-f_{l}^{0}}{f_{l}^{1}-f_{l}^{0}} & \text { if } f_{l}^{1}<f_{l}<f_{l}^{0} \\ 0 & \text { if } f_{l} \geq f_{l}^{0} .\end{cases}
$$

where the parameter values characterizing the linear membership functions are determined as $f_{1}^{1}=-131.65$, $f_{1}^{0}=-69.26, f_{2}^{1}=-93.91$, and $f_{2}^{0}=-16.95$.

For the permissible possibility levels of $h_{1}=0.7$ and $h_{2}=0.7$, the corresponding maximin problem (20) is solved through the revised GADSLPRRSU, and the obtained result is shown at the column labeled " 1 st"in Table 3. DM1 is not satisfied with this solution, but he does not desire to update $h_{l}, l=1,2$. Thus, DM1 determines the minimal satisfactory level $\hat{\delta}=0.90$ to improve $\mu_{1}\left(f_{1}\right)$ at the expense of $\mu_{2}\left(f_{2}\right)$. Furthermore, DM1 specifies the upper bound $\Delta_{\max }=0.90$ and the lower bound $\Delta_{\min }=0.60$ for the ratio of membership functions $\Delta=\mu_{2}\left(f_{2}\right) / \mu_{1}\left(f_{1}\right)$.

Table 3. Interaction process.

\begin{tabular}{ccccc}
\hline Interaction & 1 st & 2nd & 3rd & 4 th \\
\hline$\hat{\delta}$ & - & 0.900 & 0.700 & 0.800 \\
$x_{11}$ & 12 & 22 & 23 & 28 \\
$x_{12}$ & 10 & 25 & 0 & 5 \\
$x_{13}$ & 30 & 2 & 30 & 30 \\
$x_{21}$ & 0 & 1 & 7 & 6 \\
$X_{2}$ & 1 & 17 & 1 & 1 \\
$X_{2}$ & 29 & 17 & 25 & 23 \\
$\mu_{1}\left(f_{1}\right)$ & 0.661 & 0.939 & 0.731 & 0.842 \\
$\mu_{2}\left(f_{2}\right)$ & 0.667 & 0.516 & 0.662 & 0.641 \\
$\Delta$ & 1.008 & 0.549 & 0.906 & 0.762 \\
\hline
\end{tabular}


For the updated value of $\hat{\delta}$, the corresponding problem (25) is solved by the revised GADSLPRRSU. The obtained result is shown at the column labeled " 2 nd"in Table 3. Since the ratio of satisfactory degrees $\Delta$ is less than $\Delta_{\min }=0.60$, the second condition of termination of the interactive process is not fulfilled. Hence, DM1 updates the minimal satisfactory level $\hat{\delta}$ from 0.90 to 0.70 for improving $\mu_{2}\left(f_{2}\right)$ at the sacrifice of $\mu_{1}\left(f_{1}\right)$. For the updated value of $\hat{\delta}$, the corresponding (25) is solved, and the obtained result is shown at the column labeled "3rd" in Table 3. DM1 considers that $\mu_{1}\left(f_{1}\right)$ is improved but $\Delta$ is greater than $\Delta_{\max }$. Hence, DM1 is not satisfied with this solution and updates the minimal satisfactory level $\hat{\delta}$ from 0.70 to 0.80 . For the updated value of $\hat{\delta}$, the corresponding (25) is solved, and the obtained result is shown at the column labeled "4th" in Table 3. Since $\Delta$ exists in the interval $\left[\Delta_{\min }, \Delta_{\max }\right]$ and DM1 satisfied with the balance between $\mu_{1}\left(f_{1}\right)$ and $\mu_{2}\left(f_{2}\right)$, the interactive algorithm is terminated.

In the proposed interactive fuzzy nonlinear programming, through a series of update procedures of the minimal satisfactory level $\hat{\delta}$, it can be possible to obtain a satisfactory solution where the satisfactory degree of DM1 is guaranteed to be greater than or equal to the minimal satisfactory level $\hat{\delta}$ and is well balanced with that of DM2.

\section{Conclusion}

In this paper, for tackling cooperative decision making problems in hierarchical organizations under random fuzzy environments, we introduced fuzzy two-level integer programming problems involving random fuzzy variables. Considering the probabilities that the decision makers' objective function values are smaller than or equal to target variables, fuzzy goals of the decision makers for the probabilities were introduced. Through the use of fractile criteria in stochastic programming, the original random fuzzy two-level programming problems were reduced to deterministic ones. In order to obtain a satisfactory solution for the decision maker at the upper level in consideration of the cooperative relation between decision makers, interactive fuzzy programming for random fuzzy two-level integer programming problems was proposed. It was shown that all of the problems to be solved in the proposed interactive fuzzy programming can be solved through genetic algorithms for nonlinear integer programming, called the revised GADSLPRRSU. An illustrative numerical example demonstrated the feasibility and efficiency of the proposed method. Extensions to other stochastic programming models will be considered elsewhere. Also extensions to noncooperative environments will be required in the near future.

\section{REFERENCES}

[1] K. Shimizu, Y. Ishizuka and J. F. Bard, "Nondifferentiable and Two-Level Mathematical Programming," Kluwer Academic Publishers, Boston, 1997. doi:10.1007/978-1-4615-6305-1

[2] M. Sakawa and I. Nishizaki, "Cooperative and Noncooperative Multi-Level Programming," Springer, New York, 2009.

[3] W. F. Bialas and M. H. Karwan, "Two-Level Linear Programming," Management Science, Vol. 30, No. 8, 1984, pp. 1004-1020. doi:10.1287/mnsc.30.8.1004

[4] I. Nishizaki and M. Sakawa, "Computational Methods through Genetic Algorithms for Obtaining Stackelberg Solutions to Two-Level Mixed Zero-One Programming Problems," Cybernetics and Systems: An International Journal, Vol. 31, No. 2, 2000, pp. 203-221. doi:10.1080/019697200124892

[5] M. Simaan and J. B. Cruz Jr., "On the Stackelberg Strategy in Nonzero-Sum Games," Journal of Optimization Theory and Applications, Vol. 11, No. 5, 1973, pp. 533555. doi:10.1007/BF00935665

[6] Y. J. Lai, "Hierarchical Optimization: A Satisfactory Solution," Fuzzy Sets and Systems, Vol. 77, No. 3, 1996, pp. 321-325. doi:10.1016/0165-0114(95)00086-0

[7] H. S. Shih, Y. J. Lai and E. S. Lee, "Fuzzy Approach for Multi-Level Programming Problems," Computers and Operations Research, Vol. 23, No. 1, 1996, pp. 73-91. doi:10.1016/0305-0548(95)00007-9

[8] M. Sakawa, I. Nishizaki and Y. Uemura, "Interactive Fuzzy Programming for Multi-Level Linear Programming Problems," Computers \& Mathematics with Applications, Vol. 36, No. 2, 1998, pp. 71-86. doi:10.1016/S0898-1221(98)00118-7

[9] M. Sakawa, I. Nishizaki and Y. Uemura, "Interactive Fuzzy Programming for Multi-Level Linear Fractional Programming Problems with Fuzzy Parameters," Fuzzy Sets and Systems, Vol. 109, No. 1, 2000, pp. 3-19. doi:10.1016/S0165-0114(98)00130-4

[10] M. Sakawa, I. Nishizaki and Y. Uemura, "Interactive Fuzzy Programming for Two-Level Linear and Linear Fractional Production and Assignment Problems: A Case Study," European Journal of Operational Research, Vol. 135, No. 1, 2001, pp. 142-157. doi:10.1016/S0377-2217(00)00309-X

[11] M. Sakawa and I. Nishizaki, "Interactive Fuzzy Programming for Decentralized Two-Level Linear Programming Problems," Fuzzy Sets and Systems, Vol. 125, No. 3, 2002, pp. 301-315. doi:10.1016/S0165-0114(01)00042-2

[12] M. Sakawa, I. Nishizaki and Y. Uemura, "A Decentralized Two-Level Transportation Problem in a Housing Material Manufacturer: Interactive Fuzzy Programming Approach," European Journal of Operations Research, Vol. 141 , No. 1, 2002, pp. 167-185. doi:10.1016/S0377-2217(01)00273-9

[13] M. Sakawa, I. Nishizaki and Y. Uemura, "Interactive Fuzzy Programming for Two-Level Linear Fractional Programming Problems with Fuzzy Parameters," Fuzzy Sets and Systems, Vol. 115, No. 1, 2000, pp. 93-103. doi:10.1016/S0165-0114(99)00027-5 
[14] M. Sakawa and I. Nishizaki, "Interactive Fuzzy Programming for Two-Level Nonconvex Programming Problems with Fuzzy Parameters through Genetic Algorithms," Fuzzy Sets and Systems, Vol. 127, No. 2, 2002, pp. 185-197. doi:10.1016/S0165-0114(01)00134-8

[15] M. Sakawa and H. Katagiri, "Interactive Fuzzy Programming Based on Fractile Criterion Optimization Model for Two-Level Stochastic Linear Programming Problems," Cybernetics and Systems, Vol. 41, No. 7, 2010, pp. 508521. doi:10.1080/01969722.2010.511547

[16] M. Sakawa and K. Kato, "Interactive Fuzzy Programming for Stochastic Two-Level Linear Programming Problems through Probability Maximization," Interim Report, IR09-013, International Institute for Applied Systems Analysis (IIASA), 2009.

[17] M. Sakawa, H. Katagiri and T. Matsui, "Interactive Fuzzy Stochastic Two-Level Integer Programming through Fractile Criterion Optimization," Operational Research: An International Journal, Vol. 12, No. 2, 2012, pp. 209-227. doi:10.1016/S0377-2217(01)00273-9

[18] M. Sakawa, H. Katagiri and T. Matsui, "Interactive Fuzzy Random Two-Level Linear Programming through Fractile Criterion Optimization," Mathematical and Computer Modelling, Vol. 54, No. 11-12, 2011, pp. 3153-3163. doi:10.1016/j.mcm.2011.08.006

[19] M. Sakawa, I. Nishizaki and H. Katagiri, "Fuzzy Stochastic Multiobjective Programming," Springer, New York, 2011. doi:10.1007/978-1-4419-8402-9

[20] H. Kwakernaak, "Fuzzy Random Variables. I. Definitions and Theorems," Information Sciences, Vol. 15, No. 1, 1978, pp. 1-29. doi:10.1016/0020-0255(78)90019-1

[21] M. L. Puri and D. A. Ralescu, "Fuzzy Random Variables," Journal of Mathematical Analysis and Applications, Vol. 114, No. 2, 1986, pp. 409-422. doi:10.1016/0022-247X(86)90093-4

[22] G.-Y. Wang and Z. Qiao, "Linear Programming with Fuzzy Random Variable Coefficients," Fuzzy Sets and Systems, Vol. 57, No. 3, 1993, pp. 295-311. doi:10.1016/0165-0114(93)90025-D

[23] M. Sakawa and I. Nishizaki, "Interactive Fuzzy Programming for Multi-Level Programming Problems: A Re- view," International Journal of Multicriteria Decision Making, Vol. 2, No. 3, 2012, pp. 241-266. doi:10.1504/IJMCDM.2012.047846

[24] B. Liu, "Random Fuzzy Dependent-Chance Programming and Its Hybrid Intelligent Algorithm," Information Sciences, Vol. 141, No. 3-4, 2002, pp. 259-271. doi:10.1016/S0020-0255(02)00176-7

[25] M. Sakawa and T. Matsui, "Interactive Fuzzy Programming for Random Fuzzy Two-Level Programming Problems through Possibility-Based Fractile Model," Expert Systems with Applications, Vol. 39, No. 16, 2012, pp. 12599 12604. doi:10.1016/j.eswa.2012.05.024

[26] A. M. Geoffrion, "Stochastic Programming with Aspiration or Fractile Criteria," Management Science, Vol. 13, No. 9, 1967, pp. 672-679. doi:10.1287/mnsc.13.9.672

[27] M. A. Gil, M. Lopez-Diaz and D. A. Ralescu, "Overview on the Development of Fuzzy Random Variables," Fuzzy Sets and Systems, Vol. 157, No. 19, 2006, pp. 2546-2557. doi:10.1016/i.fss.2006.05.002

[28] S. Nahmias, "Fuzzy Variables," Fuzzy Sets and Systems, Vol. 1, No. 2, 1978, pp. 97-110. doi:10.1016/0165-0114(78)90011-8

[29] M. Sakawa, "Genetic Algorithms and Fuzzy Multiobjective Optimization," Kluwer Academic Publishers, Boston, 2001.

[30] S. Koziel and Z. Michalewicz, "Evolutionary Algorithms, Homomorphous Mapping, and Constrained Parameter Optimization," Evolutionary Computation, Vol. 7, No. 1, 1999, pp. 19-44. doi:10.1162/evco.1999.7.1.19

[31] Z. Michalewicz and G. Nazhiyath, "GenocopIII: A CoEvolutionary Algorithm for Numerical Optimization Problems with Nonlinear Constraints," Proceedings of the Second IEEE International Conference on Evolutionary Computation, Perth, 29 November-1 December 1995, pp. 647-651.

[32] M. Sakawa, K. Kato, M. A. K. Azad and R. Watanabe, "A Genetic Algorithm with Double String for Nonlinear Integer Programming Problems," Proceedings of 2005 IEEE International Conference on Systems, Man and Cybernetics, Waikoloa, 10-12 October 2005, pp. 3281-3286. 